\title{
Artikel
}

Werner Greve, Stefan Krankenhagen*

\section{Authentische Fiktionen. Selbst-Darstellung und Identitätskonstruktion bei William F. Cody und Karl May}


Abstract: This article deals with the cultural-historical change of public self-presentation and construction of identity at the end of the 19th century with the examples of William F. Cody ("Buffalo Bill") and Karl May. The impact of the various public, both real and virtual, stages, the change of selfrepresentation as response to public reaction, and the interaction of public and private self-perception will be examined in particular with regard to the question how authenticity and illusion are negotiated individually and socially (within the media and publicly). The importance of physical presentation (as a sign of authenticity) and the increasing necessity to claim and proof (and thus to simulate) "reality" are particular objects of study. Both sample cases, within the specific cultural-historical context of their time, demonstrate change and diversification of public self-presentations which already display in their increasing virtuosity and plurality important aspects of modern mass mediality.

Keywords: self-representation; construction of identity; William F. Cody; Karl May

*Prof. Dr. Werner Greve: Institut für Psychologie, Universität Hildesheim, Universitätsplatz 1, D-31141 Hildesheim, email: wgreve@uni-hildesheim.de

Prof. Dr. Stefan Krankenhagen, Institut für Medien und Populäre Kultur, Stiftung Universität Hildesheim, Universitätsplatz 1, D-31141, email: krankenh@uni-hildesheim.de

Es überrascht niemanden, wenn man bemerkt, dass die See an manchen Stellen tief ist und dass dort seltsame Wesen leben, die man an der offensichtlicheren Oberfläche kaum je zu Gesicht bekommt. Und doch ist es lehrreich, wenn man einzelne Exemplare dieser seltsamen Lebewesen einmal näher besieht; sie sagen einem etwas über die See, was man noch nicht wusste, und geben dem, was man schon wusste, etwas mehr Tiefenschärfe. Und so sind, obwohl es keine Neuigkeit ist festzustellen, dass das Selbst immer auch ein kulturell gemachtes Selbst ist, außergewöhnliche Fallbeispiele doch überraschend hilfreich, das Verständnis der Diversität von Selbst-Darstellung und Identitätskonstruktion ein wenig zu weiten.

Die hier besehenen beiden Fälle sind sogar in mehrfacher Hinsicht lehrreich, auch über das hinaus, was an ihnen selbst, als die Fälle, die sie sind, interessieren könnte. Selbst wenn systematische Selbstdarstellung seit Cäsars Gallischer Krieg ein wiederkehrend genutzter Modus war, ist doch das ausgehende 19. Jahrhundert, die Morgendämmerung des medialen Zeitalters, in besonderer Weise gekennzeichnet durch neue Formen, sich Bühnen zu schaffen und sich auf Bühnen zu erschaffen. ${ }^{1}$ Zwei Möglichkeiten, wie

1 Wohl um diese Zeit wird, gewiss nicht zufällig, der Begriff der Rolle eingeführt, die wir nicht spielen, sondern sind sich Selbstkonstruktion und Figurenkonstruktion überlagern können, zeigen die hier betrachteten Fälle exemplarisch, vielleicht tatsächlich prototypisch, für die sich entwickelnden populären Genres des ausgehenden 19. und beginnenden 20 . Jahrhunderts - bis heute.

Zugleich unterscheiden sie sich tiefgreifend voneinander nicht nur darin, welche Mittel angewandt werden, sondern vor allem auch darin, wen die Inszenierung adressiert. Während der eine der Welt ein Schauspiel offeriert, das sie als Sensation betrachten und deswegen glauben darf, will der andere neben aller Welt wohl auch sich selbst davon überzeugen, dass das, was da (von inm) dargestellt wird, wirklich so gewesen sein muss. Diese Differenz macht deutlich, dass jenseits der zeitgeistlichen Konvergenz und Gemeinsamkeit die individuelle psychische Ausgangsund Motivlage erheblich divergieren kann - und mit ihr der konkrete Phänotyp der Selbst-Darstellung. Die Wege, die die beiden hier betrachteten Fälle beschreiten, sind daher durchaus divergent. Umso lehrreicher könnte es sein, die gleichwohl identifizierbaren Konvergenzen und Schnittflächen näher zu besehen.

(vgl. Roselt/Weiler 2011). Er ist, seit langem, im common sense so fest verankert, dass er jede Anspielung auf das Fiktionale und Gespielte längst nicht mehr transportiert. 


\section{Vom Leben auf die Bühne und back again: Selbstdarstellung als Alltagspraxis}

Das Leben authentisch auf die geschriebene oder zu bespielende Bühne zu bringen, ist lange nicht der wesentliche Zweck öffentlicher Darstellung gewesen, Karl Ove Knausgård und anderen Meistern der literarisierten Wirklichkeit zum Trotz. Nicht in der Literatur (wenn sie Literatur sein wollte), nicht in der darstellenden Kunst, gewiss nicht im Theater. Eine Alternative zur Wirklichkeit zu sein war der Zweck der Künste, aus mancherlei Motiven. Erst an der Schwelle zur Moderne sollte Kunst (auch) zu sehen helfen, wie es (woanders, bei anderen, in anderen) tatsächlich aussah. Und je hartnäckiger dabei an der "monumentalen Selbstentblößung" (Matthias Hannemann über den letzten Roman Knausgårds) in der Darstellung festgehalten wurde, je öfter ein Ich sich wirklich darstellte, desto wahrer und wahrscheinlicher ist auch, dass diese Darstellung ein Teil dieses Ichs wird, also dessen, wovon sie handelt. Die Fälle, die im Folgenden näher untersucht werden sollen, sind für diese Form der Überblendung von Darstellung und Dargestellten besondere und besonders sprechende Beispiele auf den nicht nur je gewählten (und erzeugten) Bühnen, sondern auch auf einer zeitgeschichtlichen Bühne, die für diese neue Form der "blended identity" besonders ansprechbar war.

Dabei geht die hier stattfindende Untersuchung davon aus, dass alle Konstruktionen von Identität, wie tiefgreifend, beharrlich, eben identitätsstiftend sie auch immer operieren, immer vor dem Hintergrund einer von dieser Konstruktion unabhängigen inneren wie äußeren Welt und Wirklichkeit leben. Nicht erst die Untersuchung dieser Konstruktionen, sondern schon die Konstruktion selbst braucht unerlässlich die Idee der Wirklichkeit. Sie braucht sie, um die Authentizität des Behaupteten denken zu können - so wie die Adressaten, als Zuschauer der Selbst-Behauptung, sie nur glauben können auf der Grundlage jener Idee einer vormedialen, eben authentischen Wirklichkeit. Das heißt im Umkehrschluss allerdings auch, dass der Begriff und die Vorstellung von Authentizität erst auf der Folie ihrer Problematisierung entstehen. "Das Authentizitätsproblem kann erst dann auftauchen, wenn unter dem Bewußtsein der prinzipiellen mimetischen Differenz zwischen Darstellung und Dargestelltem
Zweifel bezüglich der Darstellungstransparenz existieren können." (Mohn/Strub/Wartemann 1997: o.S.) Die Vorstellung einer vormedialen und unvermittelten Wirklichkeit wäre somit die Grundlage, "deren konkrete Negationen die Authentizitätskonzepte im eigentlichen Sinn sind" (ebd.). So attraktiv die Dekonstruktion essentialistischer Begriffsfelder (hier: Wirklichkeit, Identität, Authentizität) auch immer sein mag - sie kann niemals die ganze Geschichte sein (und sei es nur, weil mindestens dies, dass sie die ganze Geschichte wäre, dann ja wiederum nicht konstruiert wäre).

So ist es nicht nur nicht verwunderlich, sondern beinahe zwangsläufig, dass mit der Konjunktur der Identitätskonstruktionen (die die hier zu betrachtenden Prototypen eindrucksvoll illustrieren werden) zugleich auch ihre Reflexion aufblüht. Es ist kein Zufall, dass Nietzsches berühmtes Bonmot in Jenseits von Gut und Böse (",'Das habe ich getan', sagt mein Gedächtnis. ,Das kann ich nicht getan haben' - sagt mein Stolz, und bleibt unerbittlich. Endlich - gibt das Gedächtnis nach"), in dem das Manöver der inneren Scheinwirklichkeit kritisiert und also wahrgenommen wird, in die hier betrachtete Zeit des letzten Drittels der 19. Jahrhunderts fällt. Zur Wende des Jahrhunderts wird Sigmund Freud (Die Traumdeutung erscheint 1900) dann den psychoanalytischen Blick hinter die Bühne des eigenen Bewusstseins (der wiederum auch, und unvermeidlich, den Anspruch erhebt, wahrer zu sein als das, was der Akteur oder Patient von sich selbst auf dieser Bühne erlebt) für viele Jahrzehnte zum allgemein geglaubten Denkmodell machen. ${ }^{2}$ Freuds "Raumprogramm von Couch und Sessel" (Jensen 2005: 347) war eine der vielen innovativen Bühnen einer reflexiven Selbst-Behauptung im Umbruch vom 19. auf das 20. Jahrhundert.

Doch die klassische Bühne der Selbstpräsentation, zumal der Selbst-Darstellung in anderen Identitäten als der eigenen oder eigentlichen, war und ist nicht die Couch, sondern die performativ organisierte Theaterbühne. Neu ist nicht,

2 Auch wenn diese Theorie nie konkurrenzlos war, verschwand sie erst in der zweiten Hälfte des 20. Jahrhunderts wieder aus dem Mainstream der akademischen Psychologie; in der therapeutischen Praxis und in vielen sozial- wie kulturwissenschaftlichen Ansätzen ist sie bis heute prominent. 
sie für die Erschaffung alternativer Identitäten zu nutzen (das ist natürlich ihr Zweck seit Shakespeare und Moliere gewesen), neu ist, dem, was auf der Bühne präsentiert wird, lebenspraktische Authentizität zuzuschreiben, ja dies von ihr zu fordern. Mehr noch als das Theater müssen daher populärkulturell bespielte Bühnen, wie der Zirkus etwa, behaupten, dass das, was man "wahr"nimmt, echt ist, kein Trick, der mit einer Differenz von Darstellung und Dargestelltem spielt. Hans Ulrich Gumbrecht schlägt dafür den Begriff der "Präsenzkultur" vor - im Unterschied zu einer "Sinnkultur", welche mit der hermeneutisch auszunutzenden Lücke zwischen Signifikat und Signifikant spielt: „Präsenzkultur [...] wird dominiert von den Dimensionen des Raums, denn Räume konstituieren sich um Körper - also um die zentrale menschliche Selbstreferenz der Präsenzkultur" (Gumbrecht 2001: 67). ${ }^{3}$ Letztendlich wird die Wirklichkeit der Darstellung in den Räumen des Theaters oder der Unterhaltungsbühne nicht nur behauptet, sie wird gezeigt - daher ist, was man sieht, eine echte Show als ein Präsenz erzeugendes Zeigen der Körper im Raum.

Wie jede Form der Unterhaltung, benötigt der Zirkus, die Show, die Bühne also eine ausreichende Anzahl an Authentizitätssignalen und -signets. Diese müssen sich gar nicht ausschließlich auf die hier im Fokus stehende authentische Fiktion der Selbst-Behauptung beziehen. Es reicht, wenn der Trick gelingt, wenn der Löwe durch den Reifen springt, die Akrobatin das Seil erwischt, das Messer die Scheibe (und nicht den Kopf der Assistentin) trifft. Der Moment des Gelingens ist deshalb so zentral für die Formen (und Körper) der populären Unterhaltung, weil sich darin eine praktische Schönheit ausdrückt (vgl. Hügel 2007). Das gelingende Moment verstärkt die Präsenz der Körper und mehr noch: es schafft eine Ko-Präsenz von Bühne und Zuschauersaal, von Akteuren und Zuschauerinnen, die jenen Präsenzerfahrungen zustimmen können, im Moment des eigenen Erlebens sogar zustimmen müssen. Erwischt der Körper der Akrobatin das Seil nach einem halsbrecherischen Doppelsalto ohne Boden

3 Das heißt nicht, dass Gumbrecht einer schlichten Dichotomie folgen würde. Tatsächlich ist jedes "kulturelle Dispositiv als eine je verschieden strukturierte und proportionierte Kombination aus Elementen beider Typen zu beschreiben" (Gumbrecht 2001: 66). ist es sehr schwer, nicht zu klatschen. Der eigene (Rezipienten-)Körper hat die Bewegung gleichsam mit vollzogen.

Die Formate der Populären Kultur (als auch deren unlösbare Verstrickung mit den technischen Medien der Zeit) waren Mitte des 19. Jahrhunderts in den Vereinigten Staaten deutlich weiter entwickelt als in Europa. Was etwa im Habsburger Reich als Wunderkammer des Erzherzog Ferdinands II auf Schloss Ambras in Tirol nur wenige feudale Gästen zu sehen bekamen, das wurde in New York 1841 als Barnum's American Museum schon der Öffentlichkeit präsentiert. Der spätere Zirkusdirektor P.T. Barnum stand hinter dem Konzept eines enorm publikumswirksamen Kuriositätenkabinetts zu Unterhaltungs- und Bildungszwecken einer sich rasant entwickelnden urbanen Massenkultur (vgl. Rydell/Kroes 2005). Aber es ist nicht P.T. Barnum, der hier als erste unserer beiden Fallgeschichten exemplarisch untersucht werden soll (obwohl er sich dazu durchaus eignen würde), sondern William F. Cody, der unter den Namen Buffalo Bill mit einem ebenso hybriden Unterhaltungsformat experimentierte. Seine Wild West Show integrierte dabei neben dem präsentischen Moment des Gelingens, die performativen und figurativen Elemente des Theaters, wie auch die erzählende Struktur der Historiographie.

Anfangs scheint er die Form dafür noch nicht gefunden zu haben. Für den 4. Juli 1882, als 36jähriger Familienvater wohnhaft in Nebraska, organisiert Cody zum ersten Mal eine Freiluft-Show, die, als Feier der amerikanischen Unabhängigkeit, später als Old Glory Blowout bezeichnet werden sollte. Noch später, heute sozusagen, will man darin ein Vorläufer-Format von Codys Wild West Show, aber auch die erste Aufführung einer modernen Rodeo-Show erkennen (vgl. Moses 1996). Deutlich wird, dass die Aufführung spezifischer, mit der Eroberung des Westens eng verknüpfter, körperlicher skills im Vordergrund des Programmes stand: "Cody had bucking broncos, buffalo riding, steer roping, and horse races and paid cash prizes to the participants." (Yost 2005, o.S.) Fähigkeiten der Trapper, Scouts und Cowboys, die zu diesem Zeitpunkt ihrer praktischen Aufgaben in der Eroberung des Westens historisch bereits enthoben waren und ihrer Musealisierung entgegen sahen, wurden zum zentralen Moment der Darstellung; das meint, sie wurden gezeigt, um zu gelingen. Die 
Bühne, ihre Inszenierung und ihre Akteure, senden permanente Authentizitätssignale.

Doch die Körper der Cowboys auf der Bühne des Gelingens reichten nicht aus, oder besser: das Konzept war ausbaufähig. Und so entwickelte sich das, was ab 1883 die Wild West Show heiBen sollte, unter zwei entscheidenden Prämissen: Zum einen durch den Eintritt indianischer Körper in die Show und damit durch die Dramatisierung eines Konflikts, dessen populärkulturelles Potential bis heute nicht ausgeschöpft scheint: der Kampf von Cowboys gegen Indianer. Zum anderen durch die Fiktionalisierung William F. Codys, dessen Bühnenkörper beharrlich zwischen Selbst und Figur oszillierte. Dieser zweite Punkt soll hier genauer betrachtet werden, denn es sieht so aus, als ob William F. Cody schon immer Buffalo Bill war und Buffalo Bill schon immer William F. Cody. Ein autobiographischer Bericht, den er auf der Höhe seines Ruhmes, auf der ersten EuropaStation der Show in London schrieb, ist betitelt: My First Dead Indian (vgl. Krankenhagen 2017). Diesen ersten Indianer ermordeten Cody und Buffalo Bill angeblich mit zwölf Jahren, spätestens da beginnt ihre Liaison. Im Jahr 1887, als My First Dead Indian erschien, war William F. Cody 41 Jahre alt. Er hatte bis dahin als Viehjunge gearbeitet, als Scout für die Kavallerie an der Eroberung des amerikanischen Westens teilgehabt und sich seinen Spitznamen durch die Erschießung von über 4.000 Büffeln in wenigen Monaten erworben. Aber Cody hatte zu dieser Zeit auch bereits als Schauspieler seiner eigenen Lebensgeschichte auf Bühnen der Ostküste gestanden und sein Leben als ein Stück gespielt. Die Buffalo Bill Combination gründete ihre theatralen Geschichten auf den Erlebnissen der Serienfigur Buffalo Bill, die wiederum von dem New Yorker Autor Ned Buntline seit 1869 wöchentlich in Form populärer Groschenromane veröffentlicht wurden. Mit großem Erfolg: das urbane Publikum im Osten des Landes war wild nach Wildem, war wild nach dem Wilden Westen.

Entscheidend für die Konstituierung von Buffalo William F. Cody Bill war also die temporäre Ununterscheidbarkeit von Selbst und Rolle. Das ist wichtig: Beide Kategorien müssen als eigenständige und unterscheidbare Bühnen behauptet werden. Erst auf dieser Grundlage kommt es zu jenem Oszillieren, das uns fragen lässt, welches Ich spricht, wenn es heißt: "I was twelve years old when I killed my first Indian" (Cody 1887)? William F. Cody oder Buffalo Bill? Oder eben: beide. Denn die Ununterscheidbarkeit von Selbst und Rolle ist keine mythische Konstruktion, sondern ein konstituierendes Merkmal des Stars in der Populären Kultur. William F. Cody war einer der ersten. Auch wenn sich die Inszenierungsweisen des Stars über die Zeit gewandelt haben (vgl. Museum Folkwang 2010), so gilt für Cody bereits dasselbe wie für Lady Gaga und alle anderen popmusikalischen Stars.

In der Pop-Musik wird erstens der vorher nur angedeutete Performer generisch, der niemals endgültig erkennen lässt, ob er eine Rolle spielt, einem Skript folgt oder von sich spricht und keinem Skript folgt: Pop-Musik-Performer tun immer beides und beides nie ganz. Zweitens ist Pop-Musik weder im Kinder- und Jugendzimmer noch beim Live-Konzert komplett. Sie lebt als Musik vom Wiedererkennen, aber es bleibt nicht beim Wiedererkennen einer musikalischen Gestalt, sondern es geht um das Wiedererkennen der jeweils einen Situation in der anderen. Ich kann zuhause den Rhythmus, den Sound oder die Stimme erkennen, die mir eine soziale Situation draußen, eine wahnsinnige Nacht, ein enttäuschendes Fest oder was auch immer geliefert hat. Und ich erkenne in der Öffentlichkeit, im Club oder beim Konzert die Stimme, das Lied, in das ich zuhause ungestört sehnsüchtig projiziert habe. Ich muss die Verbindung zwischen beiden aktiv herstellen. Ich muss zwischen den beiden Orten hin und herlaufen - das macht mich zum Komplizen, zum Medium. [...] Es kommt zu einem ontologischen Tauschhandel: Was ich ihnen als Publikum an Realität nachreiche, indem ich ihre Diagnose bestätigend durch die Realität laufe und Teil von ihr werde, geben sie mir als Fiktionalität, Künstlichkeit und Überhöhung zurück. (Diederichsen 2014: 249f.)

Jener ontologische Tauschhandel, jenes Bewegen zwischen den Räumen des Selbst und den Räumen der Rolle beginnt, spätestens, im Medienjahrhundert vor etwa 150 Jahren. Dadurch wird auch deutlich, wie konstituierend das Publikum für die Etablierung der Populären Kultur in dieser Zeit, und bis heute, gewesen ist. Erst der Blick macht den Star (vgl. Franck 1998). Aber auch: erst die Anwesenheit der Körper des Publikums macht den Tauschhandel zwischen fiktiver Realität der Rolle und realer Realität des Selbst möglich. Denn nur als Publikum bewegen wir uns zwischen jenen beiden Räumen, die von populären Stars am sinnfälligsten geöffnet werden, beziehungsweise: offen gehalten werden. Auf der Mikro- 
ebene der Auftritte wird dies deutlich: William F. Cody trat während seiner Show durchschnittlich vier Mal auf. Er eröffnete und er beendete die Wild West Show. Dazwischen zeigte er einmal sein Können als Kunstschütze - "Novel and extraordinary Shooting by Hon. W. F. Cody (Buffalo Bill) and Dr. W. F. Carver (Evil Spirit)" - und er verteidigte eine Gruppe Siedler beim "Attack upon the Deadwood Mail Coach" als Buffalo Bill (Cody/ Carver 1883). William F. Cody tritt in allen Momenten der Show nicht als Schauspieler auf, sondern in der Gleichzeitigkeit von Rolle und Selbst; in den Augen der Betrachter mal etwas mehr Selbst, mal etwas mehr Rolle. Immer endete die Wild West Show mit einem Auftritt des Stars zu Pferd. Er grüßte in das weite Rund von Bühne und Auditorium. ${ }^{4}$ So gab Buffalo William F. Cody Bill dem Publikum die Möglichkeit, den einen im anderen wiederzuerkennen.

Es ist nur noch ein Schritt, auf die als Bühne deklarierte Bühne zu verzichten, und gleich den Alltag des sozialen Umgangs zu inszenieren, ihn als metaphorische, dafür universelle Bühne zu nutzen. Wenig später, diesmal in Deutschland im Oktober 1906, führt der Hauptmann von Köpenick der Welt vor Augen, wie real die Inszenierung werden kann, selbst wenn man nur ein paar einfache Requisiten nutzt. ${ }^{5}$ Karl May - die zweite Fallgeschichte dieser Untersuchung - ist, wie sich zeigen soll, nicht nur die historische Vorwegnahme von Wilhelm Vogt, sondern in vielem auch eine Überbietung dieser Realitätsaufführung: bei inm folgt die (Behauptung der) Wirklichkeit der Erfindung. Folgerichtig ist der Autor der Fiktion dann auch die Figur der Fiktion, deren Authentizität behauptet wird. Freilich: auch May scheitert, unvermeidlich, daran, dass seine echte Fiktion der echten Wirklichkeit am Ende nicht gewachsen ist (wir kommen darauf zurück).

Auch Mays Scheitern wird allerdings die immer weiter fortschreitende intellektuelle Zuspitzung des Gedankens der Konstruiertheit jeder Identität nicht stoppen: am Ende wird die Fiktion schließlich zur Basis von Realitätsbehauptungen

\footnotetext{
4 Zu sehen etwa in einem undatierten Filmausschnitt. https:// www.youtube.com/watch?v=kjIH5AUglos (15.06.2017).

5 Und auch hier ist die Reflexion der Wirklichkeit auf den Fersen: Berühmt geworden ist insbesondere Zuckmayers Der Hauptmann von Köpenick von 1931: die künstlerische Darstellung einer echten Inszenierung.
}

erklärt. In der Tradition des Sozialkonstruktivismus (vgl. Mead 1934) wird, nochmals eine Generation später, Erving Goffmans These Wir alle spielen Theater (1959) diese konstruktivistische Perspektive zum Äußersten treiben. ${ }^{6}$

\section{Matrjoschka in Radebeuls Wildwest - Verstecke in Verstecken in Verstecken}

Man wird sagen dürfen, dass der Roman Old Surehand, 1894 veröffentlicht, als Schlüsselroman zum Verständnis seines Autors Karl May gelesen werden kann, als Schlüsselroman für eine ganz außergewöhnliche Identitätskonstruktion. May war, 52jährig, auf dem Höhepunkt seines Ruhms, als Old Surehand erschien. Insbesondere die Westernerzählungen, Winnetou I war 1893 erschienen, hatten sich von einzelnen Beiträgen in periodisch erscheinenden Zeitschriften (etwa im „Hausschatz", vgl. Hügel 2007a) zu auflagenstarken Romanen entwickelt (vgl. Plaul 1989). Mit dem Ruhm kam die Nachfrage, und mit ihr der Produktionsdruck. ${ }^{7}$ Und mit dem Ruhm kam die Versuchung der Köpenickiade zurück, der May schon früher erlegen war. ${ }^{8}$

Die zunächst als "Reiseromane" im Verlag Fehsenfeld, dann aber als "Reiseerzählungen" (Hügel 2007a: 207) präsentierten Abenteuer,

6 Der Sozialkonstruktivismus sollte dann, in gewisser Weise neben der psychoanalytischen Version der Identitätskonstruktion, im zwanzigsten Jahrhundert eine nicht minder dominante (nun eben:) Rolle im sozialwissenschaftlichen Diskurs spielen. Auf vielen (Wissenschafts-) Bühnen wird dieses Stück bis heute gegeben (zur Kritik vgl. Greve/Enzmann 2001).

7 Die Winnetou-Bände sind illustrativ dafür, dass nun mehr und schneller produziert werden musste: Während der Text für den ersten Band erkennbar eigens geschrieben worden war (Hügel nennt ihn Mays "Glanzstück": Hügel 2007a: 215), und daher kompositorisch einheitlich wirkt, sind die Bände II und III teilweise aus früher geschriebenen Erzählungen kompiliert. Durch die oberflächliche Adaptation handelnder Personen verloren Erzählungsteile und Charaktere ihre Pointe (die Figur des "Harry" war in einer ersten unabhängigen Geschichte "Ellen" gewesen, in die der Ich-Erzähler sich verliebt; die Attraktion der Person ist noch spürbar, aber unerklärt).

8 Tatsächlich hatte May als junger Mann verschiedentlich andere Identitäten angenommen - auch durch das entsprechende äußere Erscheinungsbild ("in Uniform") unterstrichen - und wegen Amtsanmaßung und Betrug mehrere, auch längere Haftstrafen verbüßt (vgl. Wollschläger 1976; Hermann 2000; Walther 2002; Schmiedt 2011). In der Strafhaft hatte May begonnen zu schreiben. 
häufig als Ich-Erzählung geschrieben, laden dazu ein, als Berichte realer Erlebnisse gelesen zu werden. Zahlreiche Authentizitätssignale - detailgenaue Landschaftsbeschreibungen, fremdsprachliche Wörter im laufenden Text, die in Fußnoten übersetzt werden, auch die direkte Ansprache des Lesers ("Weißt Du, lieber Leser, was ein Greenhorn ist?" - Winnetou I) - suggerieren Nähe wie Seriosität, realistische Tiefenschärfe wie Erlebnisfundierung. Mit einem Wort: sie vermitteln eine um den Autor als Subjekt jener Erfahrungen und Kenntnisse zentrierte Wahrhaftigkeit. Selbstdarstellung und Selbstbild verbanden sich zunehmend in der selbstgeschaffenen Rolle und Figur, um eigene und Publikumswünsche zu erfüllen.

Dabei spielt die Körperlichkeit des Autors in mehrfacher Hinsicht eine wichtige Rolle. Zum einen stehen körperliche Aspekte ("Schmetterhand", Winnetous schwärmerisch geschildertes Aussehen $^{9}$ ) vielfach im Zentrum der Darstellung (und Charakterisierung), zum anderen macht erst die Physis des Erlebens das Erleben endgültig real. Gleichzeitig aber muss die Authentizität zunächst in der Vorstellung des Adressaten erzeugt werden - May (der bis zur Mitte der 1890er Jahre ohne Bühne und Komparsen und Attrappen auskommt) muss dafür ausschließlich auf fiktionale Mittel setzen - bis eben das nicht mehr ausreicht.

Unter dem Druck der Nachfragen (und der ersten auf sie gegebenen Antworten) hatte May schließlich doch begonnen, seine Fiktion auch außerhalb der Romane zu materialisieren. Er ließ sich Gewehre anfertigen, die den in den Romanen detailliert geschilderten Waffen möglichst nahekamen (vgl. Klußmeier Plaul 1978; Schmiedt 2011), beantwortete schriftliche Nachfragen von (insbesondere) Leserinnen bestätigend ${ }^{10}$, und trat zuletzt auch öffentlich mit entsprechendem Anspruch auf ("Kaiserliche Hoheit, soll ich als Cow-Boy oder als Schriftsteller die Unterhaltung führen?"; vgl. Wollschläger 1976: 90; Hügel 2007a: 207). Schließlich veröffentlichte er

9 Nicht zuletzt solche Passagen hatten Arno Schmidt (1963) zu seiner psychoanalytisch inspirierten Deutung von Mays Texten als kaum kaschierte (homo-)erotische Bekenntnisse veranlasst.

10 Berühmt geworden ist der Fall, in dem er einer enthusiastischen Leserin sogar eine angebliche Haarlocke Winnetous zusandte - die sich leider als Pferdehaar herausstellte (vgl. Wollschläger 1976).
Texte, in denen der Authentizitätsanspruch einer breiten Öffentlichkeit gegenüber erhoben wurde (Erlebnisse eines Vielgereisten, von Dr. Karl May, 1896). Besonders bezeichnend im Zusammenhang unserer Untersuchung sind die zu diesem Zweck eigens erstellten Fotografien, die inn als Westmann zeigen (vgl. Klußmeier/Paul 1978: 146; Plaul 1989: 213; Schmiedt 2011: 141): das Vorbild Codys in der Kostümierung Mays ist deutlich erkennbar.

Indessen weckte die Inszenierung zunehmend Aufmerksamkeit, damit auch kritische Nachfragen - und schließlich fiel das Kartenhaus zusammen. Die Reisen hatte es nie gegeben, ${ }^{11}$ die Kompetenz (40 Sprachen) war grundlos behauptet (vgl. Wollschläger 1976: 91), sogar sein Doktortitel erfunden. In einer Reihe von aller Aussichtslosigkeit trotzenden Prozessen, die May führte, wurde die Lebenslüge gnadenlos, und gnadenlos öffentlich, demontiert. ${ }^{12}$ Schließlich blieb - nach der Lebensbeichte im Jahr 1907, in der May seine Erfindungen als insofern erlebnisbasiert bezeichnet, als sie Seelenreisen gewesen seien (vielleicht ist dies die wahrhaftigste seiner Selbstbeschreibungen) - nur der Weg in die Transzendenz offen. Jene war nicht nur immun gegen weltliche Enttarnung, sondern erschloss ihm auch, am Vorabend des Weltkriegs, ein anderes, neues Publikum; zu spät vielleicht für ein glückliches Leben, aber früh genug für ein friedvolles Lebensende.

Dies alles lag, als Old Surehand erschien, noch in jahreferner Zukunft. Und doch: der Roman enthält, aufmerksam gelesen, die ganze Tragikomödie berührend wenig verschlüsselt. Er erzählt von der Suche zweier Mitglieder einer durch ein Betrugsverbrechen (!) in alle Winde verstreuten Familie nach den anderen (einer von zwei Brüdern, die voneinander nichts wissen, sucht nach

11 Späte Reisen in den Orient und in die USA, obwohl mit demonstrativem Pomp und Postkarten in die Heimat inszeniert (vgl. Wollschläger 1976; Hügel 2007a; Schmiedt 2011), konnten natürlich nichts retten, sondern nur den Kontrast verdeutlichen - auch ihm selbst.

12 Die Wut insbesondere einiger seiner Prozessgegner ist überraschend und Mays Gefühl der Verletztheit nachvollziehbar - auch wenn er die sachliche Grundlage für alle Vorwürfe zweifellos selbst geschaffen hatte. Seine hartnäckige Verteidigung der von ihm geschaffenen Fiktionen ist insofern vielleicht nicht nur Indiz dafür, dass Selbsttäuschung unter Umständen tatsächlich den Täuscher selbst täuscht, sondern auch eine mahnende Erinnerung daran, wie sehr wir alle unsere Lebenslügen brauchen. 
Angehörigen und findet schließlich den Bruder, die gemeinsame Mutter und Tante, die Mutterschwester) und die Schuldigen. Der Ich-Erzähler begegnet innen im Lauf diverser Abenteuer (ursprünglich drei, in der heutigen Standardausgabe zwei Bände) und entschlüsselt die zahlreichen Identitätsrätsel in bester Hercule-PoirotManier in einem fulminanten Schlusskapitel.

Bemerkenswert im hier gegebenen Zusammenhang sind wenigstens zwei Punkte. Zunächst flicht May in Old Surehand erstmals Erlebnisse der eigenen Biografie in die Romanhandlung ein: die Armut der Kindheit, aber auch konkrete Details. So berichtet der "Ich"-Erzähler (Old Shatterhand) in einer von mehreren sehr persönlichen Gesprächssequenzen dem Titelhelden Old Surehand, einem der beiden Brüder, unter anderem von einer eigenen (überwundene) Augenerkrankung in der frühen Kindheit in Sachsen, über die May auch als May berichtet hat („,Mein Leben und Streben": 1910). Ob es diese Erkrankung tatsächlich gegeben hat ist, bezeichnenderweise, unklar (eher zweifelhaft: Schmiedt 2011: 29f), aber den adressierten Leser_innen, die (zumal 1894) ohnehin keine Möglichkeit der Überprüfung hatten, sollte durch diese Passagen gewiss signalisiert werden: "Ich bin es wirklich!". Folgerichtig wurde in die Erstausgabe das dritten Bandes von Old Surehand (1986) demonstrativ eine der oben angesprochenen Fotografien eingefügt (Untertitel: "Old Shatterhand (Dr. Karl May) mit Winnetous Silberbüchse"; vgl. Paul 1989: 213). ${ }^{13}$

Wenn schon dieser demonstrativ als authentisch präsentierte Auftritt des Autors im Roman auffällig ist, so ist es nochmals bemerkenswerter, dass demgegenüber fast keine der handelnden Personen im Roman die ist, die sie anfangs behaupten zu sein. Apanatschka, ein Komantschenhäuptling (der sich auch für einen Komantschen hält), ist tatsächlich der verloren geglaubte halbblütige Bruder des (ebenfalls halbblütigen) Old Surehand (der sich für einen WeiBen hält) - beider Mutter ist keine Komantschin.

13 Das Medium der Fotografie, dessen sich May und Cody so stark bedienen, hatte, solange es jung war, das besonders ausgeprägte Image (!) der Authentizität, der - im Unterschied zum gemalten Bild, zur gezeichneten Illustration - eben nicht fälschbaren Unmittelbarkeit. Der Einfluss medialer Entwicklungen auf Formen und Funktionen der Selbstdarstellung verdient freilich eine eigene Untersuchung.
Apanatschkas vermeintliche Mutter ist nicht nur ebenfalls keine Komantschin, sondern auch nicht seine Mutter, sondern beider Tante (durch den Schock des Verbrechens, das auf ihrer Hochzeit geschah, geistig umnachtet). Deren Mann ist nicht ihr Mann, auch nicht Apanatschkas Vater, sondern ein Weißer, einer der beiden Schuldigen, der die Tante und das Kind entführte und sich bei den Komantschen als "Medizinmann" versteckte, was ihm gelang, weil er ein bekannter Fälscher und Betrüger ("L'Escamoteur") war. Der andere Schuldige, der Anstifter, Drahtzieher und Haupttäter, genannt "der General", ist natürlich auch kein General (sein Titel ist erfunden!), und hat ebenfalls sein Äußeres verändert, um nicht erkannt zu werden. Die Mutter der beiden Brüder, Indianerin, verzweifelt auf der Suche nach ihren entführten Kindern, hat sich als Mann verkleidet, um unauffällig im Wilden Westen suchen zu können, wo sie die Kinder vermutet. Es ist buchstäblich keiner der, der er zu sein scheint - abgesehen vom alter ego des Autors: Old Shatterhand aka Karl May. Alle sind durch ihre Biografie in ihre neue Identität gedrängt worden, teils volens (die Bösen), teils nolens (die Mutter), teils ignorans (die Söhne, die als kleine Kinder entführt wurden), und die, die sich falsche Titel angemaßt haben (der "General", der „Medizinmann") werden schließlich ihrer gerechten (harten) Strafe zugeführt.

Es fällt schwer, nicht zu vermuten, dass die höchst komplex konstruierte Gemengelage von Selbst- und Fremdtäuschungen, die - ausgerechnet - der "Ich"-Erzähler durchschaut und zur (fast) allgemeinen Erleichterung ent-deckt, in vielem von Mays eigenen Camouflagen inspiriert ist. Auch wenn es müßig ist, sich zu fragen, wie viel davon von ihm als verschlüsselte Selbstoffenbarung bewusst intendiert ist, wird derlei jemandem, der bewusst inszenierende Fotos anfertigte, Pferdehaare verschickte und Sprachkenntnisse behauptete, die niemand haben konnte, kaum gänzlich versehentlich unterlaufen sein. Die Verzweiflung, mit der May seine Identitätskonstruktionen verteidigte, und die Hartnäckigkeit, mit der er, fast sein ganzes Leben lang, immer wieder zu neuen Konstruktionen griff, verraten vielleicht etwas über das Seelenleben eines Suchenden - aber ein Psychogramm ist hier nicht unsere Absicht. Eher schon der Hinweis darauf, dass May die Strategie der Identitätskonstruktion 
so kontinuierlich und konsistent verfolgt hat, dass sie ihrerseits als identitätskonstitutiv bezeichnet werden muss. Seine Identität wird dadurch bestimmt, dass er immer wieder in neue Rollen schlüpft und von jenem Vorgang sowohl weiß als auch nicht weiß - und das gleichzeitig auf der Bühne seiner Literatur wie auf der Bühne seines Lebens.

\section{Von der Wahrheit des Klischees und von der Zwangsläufigkeit des Darstellens}

Hätte Goffman Recht, hätten wir keinen Grund, über Mays Täuschungssalti mitleidig zu lächeln, denn wir führten sie alle selbst wieder und wieder vor. Gewiss: Cody muss, anders als May, seine Identität als Westmann nicht vortäuschen, weder vor anderen, noch vor sich selbst. Auch er aber muss sie authentisch fiktionalisieren und er tut dies in ebenso zahlreichen wie unterschiedlichen populären Formaten: als ,Museumsführer' im Westen, als Hauptfigur eines Groschenromans, als Theaterbühnenfigur, als Open-Air-Impresario und Darsteller, als Werbefigur, als Postkarte und Plakat, als Filmfigur. So wird auch aus Cody eine auf Dauer gestellte Ununterscheidbarkeit zwischen personalem und performativem Selbst. Darin trifft er sich schließlich doch mit May, auch wenn er gewissermaßen aus der Gegenrichtung des tatsächlich im Westen handelnden Mannes auf die Bühne tritt, auf der Westmänner sich inszenieren.

Umso auffälliger ist es, dass es eine unübersehbare Kontinuität der Ikonographie des echten Westmannes gibt. Sie geht von General Custer zu William F. Cody zu Karl May. Und sie benötigt, als eine innovative Bildstrategie des ausgehenden 19. Jahrhunderts, die Ko-Präsenz indianischer Akteure. Für Cody ist Custer so etwas wie eine Blaupause, auf die sich sämtliche seiner Darstellungsmodi stützen: Noch vor der Inthronisierung der Wild West Show, schreibt er für seine theatralen Bühnenshows das dramatische Gedicht Custer's Death. Er nimmt, als ehemaliger Scout der Kavallerie, an Vergeltungskämpfen gegen die Cheyenne-Indianer teil und nimmt dabei The First Scalp for Custer. Jenes Motiv wiederum, als ein posthumes Erbe von Custer an Cody inszeniert, wird als Element der Wild West Show aufgenommen, kursiert als Druck, Zeichnung oder Postkarte, fungiert als Beigabe und
Souvenir und kommt als Szene im ersten Film von Cody zu (keiner) Berühmtheit. ${ }^{14}$ Schließlich: alle Abbildungen Codys imitieren von Beginn seiner Bühnenkarriere an die bestehenden bildlichen Abbildungen von Custer, indem er sich vergleichbar mit Bart, gelocktem Haar und Hut darstellen lässt. Einzig die auffällige Präsenz von Sitting Bull als Teil eines Doppelporträts des Westmanns als gleichzeitig Feind und Freund der Indianer unterscheidet die Bildstrategien Codys von denjenigen Custers (abgesehen davon, dass sie sich quantitativ ins Unermessliche steigern). ${ }^{15}$

Es ist aus mehreren Gründen bezeichnend, dass Karl May genau jene Ikonographie aufnimmt. Ähnlich wie Custer/Cody inszeniert er sich mit Schnurrbart, Hut und Büchse (vgl. Plaul 1989: 213; Schmiedt 2011: 141); ähnlich wie Cody legitimiert er seine (literarischen) Darstellungen über die Grenze von Zivilisation und Wildnis mit dem ikonisch gewordenen Doppelporträt Shatterhand/Winnetou. ${ }^{16}$ Die Ähnlich der von May eigens hergestellten Photographien zu Codys Selbstdarstellung ist frappierend. ${ }^{17}$ Aber wie immer die historisch-biografische Frage zu beantworten ist, ob May Codys Bilder tatsächlich vor Augen hatte ${ }^{18}$ bemerkenswert ist allemal, dass die fik-

14 Auch The first Scalp for Custer wird damit zu einem seriellen Motiv im Medienkosmos des Buffalo Bill. Eine Beschreibung und Illustration der Szene findet sich in Codys Autobiographie von 1879; und auch als Szene in den Kurzfilmen The Indian Wars, die Cody nach dem Bankrott der Wild West Show 1913 mit der eigenen Produktionsfirma dreht, sieht man Cody in Siegerpose über einem toten Indianer stehen, einen Skalp in seinen Händen. Paul Hedren (1987) hat jenseits dieser Mediengeschichte versucht, das Ereignis und seine Erzählung zu sortieren.

15 Das unterstreicht nochmals die besondere Bedeutung der je modernen Medien (hier: der Fotografie) für die öffentliche Inszenierung.

$\mathbf{1 6}$ Vor allem in der Nachkriegstradition ist diese Darstellung (durch die Schauspieler Lex Barker und Pierre Brice) die dominante Karl-May Ikonografie in der BRD geworden. 17 Obwohl es nicht belegt ist, spricht einiges dafür, dass May Codys Show auf ihrer Tournee durch Deutschland 1896 in Dresden gesehen hat; mindestens dürfte er über sie gelesen haben. In gewohnt selbstsicherer Manier schrieb er bereits 1894 an eine Leserin: „Buffalo Bill kenne ich persönlich". Zitiert nach: Arentz 1995: 4.

18 Der einhundertste Todestag Codys war der Hannoverschen Allgemeinen Zeitung (7.1.2017) einen ganzseitigen Beitrag wert, der in einem Exkurs die Frage der möglichen persönlichen Begegnung von May und Cody (anlässlich der Deutschlandtournee des letzteren) ausführlich - und skeptisch - diskutierte. 
tionale Selbstdarstellung des einen die Folie für die Authentizitätsfiktion des anderen wird: Gewiss nicht zuletzt die Ähnlichkeit der Bilder zu Codys (und Custers) Darstellungen machen May zunächst glaubhaft. Mit jenen medialen Bildstrategien der Ent-Körperung (oder Um-Körperung) geht die Selbst-Präsentation als Identitätskonstruktion schließlich in Serie. "[Buffalo Bill] capitalized on our modern talent for the mimetic - our ability to create countless mass-produced imitations of an original." (White 1994: 29)

Vielleicht sind Cody und May deshalb Prototypen eines beginnenden Zeitalters der universellen öffentlichen Bühne. Obwohl es immer schon auch jenseits des Theaters öffentliche Bühnen gegeben hat, auf denen sich die, die wahrgenommen werden wollten, präsentierten (die Rednerbühne des Forum Romanum, die entscheidender noch als der militärische Erfolg das Eingangstor zur politischen Karriere war), so waren diese Bühnen doch fast immer für fast alle Menschen unbetretbar. Jene Räume waren durch Tabus (Schauspieler hatten nicht nur in Rom moralisch zweifelhaften Halbweltstatus) und Gelegenheitshürden geschützt (abgesehen von Speakers Corner im Hyde Park sind die Chancen für öffentliche Reden für Jederman rar). Erst die durchgreifend egalitäre Demokratisierung moderner Gesellschaften (prototypisch in den USA), die explosionsartige Verbreitung und allgemeine Zugänglichkeit von Massenmedien (und also Öffentlichkeit), aber auch die Verdichtung von immer mehr Menschen auf immer engerem Raum (Großstädte und Ballungszentren) ändern dies grundlegend. Während die Beobachtung, unter der alle stehen, in der kleinen dörflichen (oder nomadischen) Gemeinschaft archaischer und antiker Gesellschaften, in der jede jeden kennt, vor allem der sozialen Kontrolle seiner Mitglieder dient (die Exklusion des egoistischen Täuschers und Ausbeuters von Solidarität dürfte eine wesentliche Funktion der öffentlichen Sozialkontrolle gewesen sein), verkehrt sich dies in der anonymen Massenwelt ins Gegenteil. Niemand kennt jemanden wider - es sei denn, er hat einen großen Auftritt. Zu ihm aber haben nun auch Personen Gelegenheit, die früher Niemand geblieben wären: Büffeljäger und Kleinkriminelle, beispielsweise.

Mit der Zahl der Bühnen steigt auch die Vielfalt der Rollen. Wenn schon der Bühnenstar vor der Moderne die höchst seltene Ausnahme war, war die Chance, auf anderen Bühnen jene Berühmtheit zu erringen, die nur auf sich verweist, also nur öffentliche Wahrnehmung ausdrückt, nicht auf einer unabhängig zu evaluierenden (politische, militärische, wirtschaftliche) Leistung beruht, nie zuvor gegeben. Erst eine Welt der Massen und Massenmedien macht Sichtbarkeit zu einer eigenen Währung. Wenn sie erst etabliert ist, eröffnet die Bühnenpluralität deshalb einer zunehmend fluiden Öffentlichkeit einen beinahe unbegrenzten Möglichkeitsraum der Darstellungen und des Darstellens. Wenn eine Wild-West-Show in den USA noch Folklorekolorit, beinahe Konnotation der Heimatkunde haben mag, ist der Aufstieg eines Lehrers aus Sachsen zum Westernstar in Deutschland so verblüffend wie innovativ.

Zugleich führt Mays Berühmtheit (mehr noch als die Codys) vor Augen, dass die Darstellung auf Bühnen in dem Sinne, transparent' bleiben muss, dass sie eben dies konstitutive Moment, dass sie Darstellung ist (und nicht die dargestellte Wirklichkeit), nicht selbst mit darstellen (oder in die Darstellung aufnehmen) darf. ${ }^{19}$ Die unübertreffliche Darstellung dieses kategorischen Imperativs der Darstellung ist Oscar Wildes Bildnis des Dorian Grey (1891 in der Buchfassung veröffentlicht), das dem Dargestellten jede Sünde vergibt, nur die eine nicht: dass er als Darstellung (Bildnis) betrachtet wird. Im Moment seiner Enthüllung wird der ,wirkliche' Dorian (vermeintlich außerhalb des Bildes) als bloße Fiktion erkennbar. Nach der An- und also Einsicht erinnert nur noch die im Bild dargestellte Widergabe an die Darstellung, die nun enthüllte Wirklichkeit ist dagegen abstoßend: Realität - oder Authentizität - wird zur Höchststrafe für die Hybris, hinter die Schleier der Darstellung gesehen zu haben. ${ }^{20}$ Dorian Grey, so lernen wir, muss im Modus der Pose bleiben,

19 Das wird sich erst in der darstellenden Kunst (Theater, Film, Bildende Kunst) der Nachkriegsgesellschaft Mitte des 20. Jhdt. ändern, in der der Umstand, dass die Darstellung Darstellung ist, selbst Teil der Darstellung wird: etwa in den monochromen Bildkompositionen von Yves Klein, den selbstreferentiellen Filmen Andy Warhols oder, später, den fotografischen Strategien von Cindy Sherman und Jeff Wall.

20 Dieses Motiv ist freilich älter: König Ödipus' eigentlicher Frevel (bei Sophokles) ist nicht der Vatermord und die Mutterehe (die beide unwissentlich begangen wurden), sondern der ruchlose eitle Versuch, die Rätsel der Vergangenheit gegen jede Warnung zu lösen. Folgerichtig blendet sich Ödipus zur Strafe für dieses Vergehen (Du sollst nicht 
darf die "konstitutive Offenheit zwischen Rolle und Selbst" nicht schließen wollen, wie es Diedrich Diederichsen am Beispiel des Popstars formuliert: "Gegen den Voyeurismus hilft nur eines - sich beobachten lassen." (Diederichsen 2014: 250) Die Pose (man denke an alle Fotografien Oscar Wildes), ist das zum Bild gewordene Spiel zwischen Rolle und Selbst, ist "etwas, das nicht einfach nur in der Mitte zwischen Aktion und Passion steht, sondern konkreter ein aktives Passivsein, aktives Zeigen ansteuert" (ebd.).

So gehört zur Darstellung unvermeidlich die (Fiktion der) Authentizität - und umgekehrt. Schon die Idee der Darstellung verweist darauf, dass etwas dargestellt wird, dass es auch unabhängig von der Darstellung gibt. ${ }^{21}$ Die Vorführung eines Selbst muss also zeigen, was es wirklich gibt - sie ist gerade kein Schau-Spiel, sondern immer auch Widergabe von Wirklichkeit, kein Theater, sondern Ver-Körperung. Wird das wechselseitige Spiel zwischen Selbst und Rolle infrage gestellt, implodiert die populärkulturelle Darstellung, sie wird mindestens uninteressant, womöglich ärgerlich - auch dafür ist der Fall May exemplarisch. Nicht nur die mitleid- und verständnislose Wut, mit der Teile der Öffentlichkeit auf die Enthüllung seiner Camouflage reagieren, sondern mehr noch seine posthume Entsorgung in der Kinder- und Jugendliteratur ${ }^{22}$ zeigen, dass der Weg zurück auf die Bühne der öffentlichen Wahrnehmung end-

sehen!), während seine Mutter sich als Sühne für den versuchten Sohnesmord selbst tötet (Greve, 2016).

21 Wolfgang Iser formuliert dieses im Hinblick auf Inszenierungen: "Nun beinhaltet Inszenierung, daß ihr etwas vorausliegen muß, welches durch sie zur Erscheinung kommt. Dieses Vorausliegende vermag niemals vollkommen in Inszenierung einzugehen, weil sonst dieses selbst das ihr Vorausliegende wäre. Anders gewendet ließe sich auch sagen, daß jede Inszenierung aus dem lebt, was sie nicht ist." (Iser 1993: 511)

22 Das gilt auch für jüngere Reanimationen Karl Mays. Die erfolgreiche Filmserie der 1960er Jahre konnte eben auch nur in einem Deutschland funktionieren, in dem Väter sentimental ihren Jugendidolen zusahen, wie sie auf echten Pferden durch echte Landschaften (leider in Jugoslawien aber sieht das nicht wirklich ,täuschend echt' aus?) ritten, und ihre Kinder eben glauben durften, dass dies wirklich Winnetou war, von dem sie kurz zuvor gelesen hatten. Die Karl-May-Spiele in Bad Segeberg und Elspe schöpften ihre Attraktivität dann nicht nur aus diesem Effekt, sondern aus einer bezeichnenden Authentizität zweiter Ordnung - dadurch dass Pierre Brice, der charismatische Darsteller der Winnetou-Filme, auch auf diesen Bühnen den Winnetou gültig verschlossen war. Dort darf nur auftreten, wem man den inszenierten Auftritt glauben kann.

\section{Münchhausens Ausweg: Das Recht auf Täuschung}

Man kann diesen Befund aus wenigstens zwei Perspektiven lesen: aus einer individuellen ebenso wie aus einer kulturellen Perspektive. Auf der kulturellen Ebene ist die Interpenetration von Authentizität und Fiktion spätestens in der Presentation of Self in Everyday Life (Goffman 1959) konstitutiv geworden. ${ }^{23}$ Wir leben schon lange in einer Kultur des Verdachts, in der alles eigentlich ganz anders ist, in der hinter jeder Darstellung eine andere, wirklichere Wirklichkeit lauert (vgl. Groys 2000). Aber so sehr die Darstellung und damit zugleich die Allgegenwart des Fiktiven in der Moderne publik geworden sind, so unabdingbar ist dabei die Behauptung von Authentizität geblieben. Vielleicht wird man sagen dürfen, sie habe eben wegen der Vielzahl und wachsenden Perfektion der Bühnen an Bedeutung, an Unverzichtbarkeit eher noch gewonnen, eben weil sie als Problematik sichtbar(er) wurde.

Die Bühne des Sports (prototypisch die Renaissance der Olympischen Spiele eben zu dieser Zeit, Ende des 19. Jahrhunderts) ist das vielleicht am hartnäckigsten verteidigte Naturschutzgebiet der Authentizität: Bis heute wird im Sport die (Aufdeckung der) Vortäuschung eigener Leistung (Doping) wütend geahndet und geächtet. Ebenso muss die darstellende Kunst ,live' sein, am besten , unplugged' - wer Playback nutzt

gab, konnten Kinder mit Fug und Recht sagen, sie hätten dort den, echten Winnetou' live gesehen.

23 Es ist hier nicht der Ort, die These der zeitlosen Universalität dieser gegenseitigen Durchdringung zu diskutieren. Selbstverständlich lautet die Behauptung (wenigstens einiger Varianten) des Sozialkonstruktivismus, dass wir immer schon unsere Rollen sind, dass sie - und ihre Wahrnehmung durch andere - allererst konstituieren, wer wir sind (für Ramses, Cicero und Napoleon nicht weniger als für Brad Pitt und Helmut Schmidt). Diese These verdient eine andere (und erfordert eine längere) Untersuchung (zu einigen Argumenten vgl. Greve/Enzmann 2001). Mit der (insbesondere durch die allgemeine Verfügbarkeit der modernen Medien) seit dem Ende des 19. Jahrhunderts aufgetretene Vervielfachung von öffentlichen Bühnen und Darstellungen wurde eine neue Dynamik dieser Verflechtung ermöglicht und realisiert - für die Cody und May exemplarisch, vielleicht prototypisch stehen. 
(lies: braucht), macht sich lächerlich. Selbst Filmschauspieler, deren Beruf die Darstellung ist und demonstrativ nicht die dargestellte Person sein wollen, müssen sich, um glaubhaft zu bleiben, in Cannes oder bei den Academy Awards auf dem roten Teppich zeigen: Dann kann man sehen (und bewerten), ob ihre Körper wirklich so aussehen wie auf der Leinwand. Und so braucht auch die Wild West Show echte Indianer und einen wirklichen Westmann als Star, und allemal muss die vorgeführte Artistik (Reiten, Schießen) live und also in Anwesenheit aller Körper bewertbar sein. Es kommt zu jenem ontologischen Tauschhandel zwischen Rolle und Selbst - genauer: zu einem Tauschhandel zwischen populärkulturellen Akteuren und ihrem Publikum: "Was ich ihnen als Publikum an Realität nachreiche, indem ich ihre Diagnose bestätigend durch die Realität laufe und Teil von ihr werde, geben sie mir als Fiktionalität, Künstlichkeit und Überhöhung zurück." (Diederichsen 2014: 250)

Vielleicht ist die kulturelle Täuschung hier auf vergleichbare Weise wirksam wie bei einer individuellen Selbsttäuschung: Der Getäuschte weiß um die Täuschung, aber glaubt sie dennoch, weil er an sie glauben will, weil er von der Täuschung profitiert. Wie aber profitiert man individuell von einer Selbst-Täuschung? Dave Hickey spricht von dem individuellen „Recht verführt zu werden". Denn:

Uns vor Verführung zu schützen bedeutet, uns unsere verbrieften Rechte als moralische Akteure zu verwehren. Wer unsere Begeisterung als bloßes Fan-Gebaren abtut, versteht nicht, um was [es] im Wesentlichen geht, weil die Frage, was eine beliebige Gruppe von Bürgern will, in einer freien Gesellschaft immer eine Angelegenheit ist, die politische Konsequenzen hat. (Hickey 2015: 109)

Der individuelle Profit, der Mehrwert, läge somit im Selbst-bewussten Umgang mit kulturellen Täuschungen, in der Möglichkeit - und Fähigkeit - das Spiel zwischen Selbst und Rolle zu erkennen, zu bewerten, zu liken oder zu disliken. In diesem Sinne verlangt eine moderne Massenmediengesellschaft heute nach möglichst komplexen, überraschenden, oszillierenden Varianten - und Weisen - der Selbst-Darstellung, wie sie William F. Cody und Karl May mit variierendem Erfolg (unter variierenden Perspektiven und auf variierende Publika hin) im 19. Jahrhundert entworfen haben.

\section{Literaturverzeichnis}

Arentz, Regina (1995): 113 Jahre Mythen aus dem Wilden Westen - Buffalo Bill's Wild West Show 1883-1995 und das europäische Bild des Westens. In: Karl May \& Co, Nr. 61 (Juli), S. 3-7.

Cody, William F. (1887): My First Dead Indian. In: Daily Alta California, 19.06.1887, http://codyarchive.org/ texts/wfc.nsp05848.html (15.06.2017).

Cody, William F./Carver, W.F. (1883): The Wild West Programm 1883. http://codyarchive.org/images/view/ posters/wfc.mem00271 (15.06.2017).

Diederichsen, Diedrich (2014): Olympier und Fans, Rezipienten und Götter. Menschen als Medien in verschiedenen Stadien der Kulturindustrie. In: Widmer, Ruedi (Hg.): Laienherrschaft. 18 Exkurse zum Verhältnis von Künstlern und Medien. Berlin: Diaphanes, S. 241-252.

Franck, Georg (1998): Ökonomie der Aufmerksamkeit. Ein Entwurf. München: Hanser.

Greve, Werner (2016): Der stille Triumph des Ödipus - Ein unbeachteter Sieg über das Verhängnis. In: Coincidentia 7/2, S. 161-180.

Greve, Werner/Enzmann, Dirk (2001): Etikettierungen im Jugendstrafvollzug? Wider einige Gewißheiten des Labeling-Ansatzes. In: Bereswill, Mechthild/Greve, Werner (Hgg.): Forschungsthema Strafvollzug. Baden-Baden: Nomos, S. 207-250.

Goffman, Erving (2003) [1959]: Wir alle spielen Theater. Die Selbstdarstellung im Alltag. München: Piper.

Groys, Boris (2000): Unter Verdacht. Eine Phänomenologie der Medien. München/Wien: Hanser.

Gumbrecht, Hans Ulrich (2001): Produktion von Präsenz, durchsetzt mit Absenz. Über Musik, Libretto und Inszenierung. In: Früchtl, Joseph/Zimmermann, Jörg (Hgg.): Ästhetik der Inszenierung. Frankfurt a.M.: Suhrkamp, S. 63-76.

Halbig, Matthias (07.01.2017): Schön war sie, die Prärie. In: Hannoversche Allgemeine Zeitung, S. 32.

Hannemann, Matthias (17.06.2017): Entweder hält es, oder es zerbricht. In: Frankfurter Allgemeine Zeitung.

Hickey, Dave (2015): Der unsichtbare Drache. Berlin: Merve.

Hedren, Paul (1987): First Scalp for Custer. The Skirmish at Warbonnet Creek, Nebraska, July 17, 1876. Lincoln, Nebraska: University of Nebraska Press.

Hermann, Frederik (2000): Old Shatterhand, das bin ich. Weinheim: Beltz.

Hügel, Hans-Otto (2007): Nachrichten aus dem gelingenden Leben. Die Schönheit des Populären und sein Verhältnis zur Kunst. In: ders.: Lob des Mainstreams. Zu Begriff und Geschichte von Unterhaltung und Populärer Kultur. Köln: Herbert von Halem, S. 110-147.

Hügel, Hans-Otto (2007a): Karl May. Das inszenierte Abentuer. In: ders.: Lob des Mainstreams. Zu Begriff und Geschichte von Unterhaltung und Populärer Kultur. Köln: Herbert von Halem, S. 206-224. 
Iser, Wolfgang (1993): Das Fiktive und das Imaginäre. Frankfurt a.M.: Suhrkamp.

Jensen, Uffa (2005): Die Couch. In: Geisthövel, Alexa/Knoch, Habbo (Hgg.): Orte der Moderne. Erfahrungswelten des 19. und 20. Jahrhunderts. Frankfurt a.M.: Campus, S. 345-354.

Klußmeier, Gerhard/Plaul, Hainer (1992): Karl May, das bewegte Leben eines Außenseiters. Eine Bilddokumentation. München: Heyne.

Krankenhagen, Stefan (2017): Ein Pre-Enactement. Zu William F. Cody und der Verfügbarkeit von Geschichte in der Populären Kultur. In: ders./Vahrson, Viola (Hgg.): Geschichte kuratieren. Kultur- und kunstwissenschaftliche An-Ordnungen der Vergangenheit. Köln/Weimar/Wien: Böhlau, S. 33-43.

Mead, George Herbert (1968) [1934]: Geist, Identität und Gesellschaft aus der Sicht des Sozialbehaviorismus. Frankfurt a.M.: Suhrkamp.

Mohn Elisabeth/Strub, Christian/Wartemann, Geesche (1997): Authentizität: Konzepte - Strategien - Gegenstandsfelder. Manuskript, Universität Hildesheim, o.S.

Moses, Lester George (1996): Wild West Shows and the Image of American Indians 1883-1933. Albuquerque: University of New Mexico Press.
Museum Folkwang Essen (Hg.) (2010): A Star is born Fotografie und Rock seit Elvis. Göttingen.

Plaul, Hainer (1989): Illustrierte Karl May Bibliographie. München: Saur.

Roselt, Jens/Weiler, Christel (Hgg.) (2011): Schauspielen heute. Die Bildung des Menschen in den performativen Künsten. Bielefeld: transcript.

Rydell, Robert W./Kroes, Rob (2005): Buffalo Bill in Bologna: The Americanization of the World, 1869-1922. Chicago: University of Chicago Press.

Schmiedt, Helmut (2011): Karl May oder die Macht der Phantasie. München: Beck.

Schmidt, Arno (1963): Sitara und der Weg dorthin. Eine Studie über Wesen, Werk \& Wirkung Karl Mays. Stahlberg: Karlsruhe.

Walther, Klaus (2002): Karl May. München: dtv.

White, Richard (1994): Frederick Jackson Turner and Buffalo Bill. In: ders./Nelson Limerick, Patricia: The Frontier in American Culture. Berkeley/Los Angeles: Univ. of California Press, S. 6-65.

Wollschläger, Hans (1976): Karl May. München: Diogenes. Yost, Nellie (2005): North Platte - Lincoln County. http:// casde.unl.edu/history/counties/lincoln/northplatte/ (15.06.2017). 